\title{
Modal Kinetic Energy Change Ratio-Based Damage Assessment of Laminated Composite BEAMS USING NoISY AND INCOMPLETE MEASUREMENTS
}

\author{
Du DINH-CONG ${ }^{1,3, *}$, Linh VO-VAN $N^{3}$, Dung NGUYEN-QUOC ${ }^{3}$, Trung \\ NGUYEN-THOI ${ }^{2,3}$
}

\author{
${ }^{1}$ Division of Construction Computation, Institute for Computational Science, \\ Ton Duc Thang University, Ho Chi Minh City, Vietnam \\ ${ }^{2}$ Division of Computational Mathematics and Engineering, Institute for Computational Science, \\ Ton Duc Thang University, Ho Chi Minh City, Vietnam \\ ${ }^{3}$ Faculty of Civil Engineering, Ton Duc Thang University, Ho Chi Minh City, Vietnam \\ *Corresponding Author: Du Dinh-Cong (Email: dinhcongdu@tdtu.edu.vn) \\ (Received: 4-Sep-2019; accepted: 17-Sep-2019; published: 30-Sep-2019) \\ DOI: http://dx.doi.org/10.25073/jaec.201933.248
}

\begin{abstract}
Modal kinetic energy (MKE) feature has been mostly employed for optimal sensor layout strategies; nevertheless, little attention is paid to use the feature to the field of structural damage detection. The article presents the extensive applicability of $M K E$ change ratio (MKECR), a good damage sensitive parameter, to damage localization and quantification of laminated composite beams. The formulation of the parameter is based on the closed-form of element MKE sensitivity. The performance of the offered damage detection method is numerically verified by a clamped-clamped composite beam and a twospan continuous composite beam with different hypothetical damage scenarios. The influence of incomplete mode shapes, various noise levels as well as damage magnitudes on damage prediction results are also investigated. The obtained results from these numerical examples indicate that the offered method reliably localize the actual damaged elements and approximately estimate their severities, even under incomplete measurements at a high noise level.
\end{abstract}

\section{Keywords}

Damage Assessment, Modal Kinetic Energy, Modal Strain Energy, Composite Beam, Incomplete Measurements.

\section{Introduction}

Advanced composite materials possessing superior mechanical properties are finding increasing applications in different industrial sectors such as mechanics, civil, marine aeronautical and aerospace industries. In the industrial fields, composite beam-like structures have been widely utilized among various kinds of structures. Under excessive environmental and operational conditions, the composite structures may be severely deteriorated in an unexpected manner. This adversely affects its structural performance and can gradually lead to a failure mechanism. Therefore, condition monitoring and fault detection play a crucial role in maintaining the serviceability and safety of the composite beam structures. 
During the past decades, substantial research efforts have been devoted to the development of damage diagnosis techniques for composite structures with both simulation and experimental studies. Based on the comprehensive literature reviews with particular on composite materials $[1,2]$, vibration-based damage identification (VBDI) approaches have attracted substantial interest in the scientific community. Seeking or evolving damage features which are sufficiently sensitive to local damage but insensitive to modeling errors and noise contamination, is the core part of VBDI approaches. In the last years, many researchers have proposed numerous methods for damage detection, localization and estimation in composite structures using vibration parameters comprising natural frequencies $[3,4]$, flexibility matrix $[5,6]$ and modal strain energy $[7,8]$.

Among various vibration parameters, modal strain energy (MSE) derived from eigenvectors and stiffness matrix has been recognized as a sensitive indicator for the detection of local structural damage, which has been received much attention. According to comparative studies [9]- [11], it was found that MSE-based damage detection methods performed better than others. Up to now, many MSE based-damage indicators have been explored by various authors. Representative examples include Stubbs damage index (SDI) $[12,13]$, 'Modal Strain Energy Change Ratio' (MSECR) [14], cross-modal strain energy (CMSE) [15], modal strain energy equivalence index (MSEEI) [16], modal strain energy based-index (MSEBI) [17] and total modal energy index (TMEI) [18]. Several successful applications of these indicators have also been introduced on composite structures. For instance, Hu et al. [19] utilized SDI to localize surface crack damage in composite laminated plates. Pradeep et al. [20] utilized MSECR to indicate damage locations in sandwich structures. Vo-Duy et al. [21] also used MSECR in the first step of two-step damage identification procedure for finding suspected damage elements of a composite sandwich plate. Dinh-Cong et al [7] proposed a normalized MSE-based damage index (nMSEBI), an improved version of the MSEBI, to localize possible damage sites in a composite plate structure.
The above studies [7], [19]- [21] were limited to the requirement of complete model data corresponding to every degree of freedom (DOF) in a finite element model of monitored structure. In addition to this, most of these aforementioned damage indices can successfully predict damage locations, but cannot provide an approximate estimation of the corresponding damage severities. Therefore, it will be certainly desirable to present a damage index that could not only effectively locate but also quantify structural damage simultaneously. For this purpose, several methods based sensitivity analysis of dynamic parameters have been developed by some investigators [22]- [24]. Among all of the developed methods, modal kinetic energy change ratio (MKECR), recently proposed by the authors in [24], was taken as a damage sensitive indicator which can indicate both damage positions and corresponding severities. The MKECR was later utilized extensively by Dinh-Cong et al. [25] for damage localization of $2 \mathrm{D}$ frame and truss structures. In these studies [24, 25], however, the feasibility and effectiveness of the MKECR were only verified by isotropic structures.

The research work reported here is essentially an extension of MKECR to damage localization and quantification of laminated composite beams. Numerical simulations of a clampedclamped composite beam and a two-span continuous composite beam are studied to evaluate the performance of the proposed damage assessment method. The effects of incomplete mode shapes, various noise levels as well as damage magnitudes on damage detection results are also investigated to show how well the proposed method under operational conditions.

\section{MODAL KINETIC ENERGY CHANGE RATIO-BASED METHOD}

In the literature, little attention is paid to utilize modal kinetic energy (MKE) to the field of structural damage detection. Recently, Shahri and Ghorbani-Tanha introduced modal kinetic en- 
ergy change ratio (MKECR) [24] as a good damage sensitive parameter. Although this damage indicator was investigated in quantifying the damages of isotropic beam structures with numerical and experimental tests, it is necessary to explore its performance for anisotropic composite beams. In the following two subsections, a brief description of the formulation of MKECR is provided.

\subsection{Modal kinetic energy}

For a linear, undamped discrete structural system, the dynamic eigenvalue equation can be expressed as

$$
\mathbf{K} \Phi_{r}=\lambda_{r} \mathbf{M} \Phi_{r}
$$

where $\lambda_{r}$ and $\Phi_{r}$ are the $r$-th eigenvalue and eigenvector, respectively. The global stiffness matrix $\mathbf{K}$ and mass $\mathbf{M}$ are constructed by assembling their individual element stiffness matrices as

$$
\mathbf{K}=\sum_{e=1}^{N e} \mathbf{K}_{e} ; \quad \mathbf{M}=\sum_{e=1}^{N e} \mathbf{M}_{e}
$$

where $\mathbf{K}_{e}$ and $\mathbf{M}_{e}$ represent the $e$-th element stiffness and $e$-th element mass matrices of the structure, respectively.

From the definition of MKE in dynamics, the MKE values can be obtained by the combination of eigenvalues, eigenvectors and stiffness matrix [26]. For the $r$-th order mode of vibration, the $e$-th element's MKE of the intact and damaged structures are, respectively, calculated as [26]

$$
\begin{aligned}
& \mathrm{MKE}_{e r}=\frac{1}{2} \lambda_{r}\left(\mathbf{\Phi}_{r}\right)^{\mathrm{T}} \mathbf{M}_{e} \boldsymbol{\Phi}_{r}, \\
& e=(1,2, \ldots, \text { nele }) ; r=(1,2, \ldots, \text { nmod }) \\
& \mathrm{MKE}_{e r}^{d}=\frac{1}{2} \lambda_{r}\left(\boldsymbol{\Phi}_{r}^{d}\right)^{\mathrm{T}} \mathbf{M}_{e} \boldsymbol{\Phi}_{r}^{d}, \\
& \quad e=(1,2, \ldots, \text { nele }) ; r=(1,2, \ldots, \text { nmod })
\end{aligned}
$$

in which nmod is the number of considered mode shapes; and the superscripts $d$ denotes the damaged states.

It should be mentioned that in most practical situations, since only the spatially-incomplete mode shapes of damaged structure are extracted from experimental data, model reduction techniques like improved reduced system (IRS) [27, 28] or iterated IRS (IIRS) [5, 29, 30] could be utilized to handle with the issue. In this present work, the IIRS technique is adopted as follows:

$$
\boldsymbol{\Phi}_{r}^{d}=\left\{\begin{array}{c}
\boldsymbol{\Phi}_{r, m}^{d} \\
\boldsymbol{\Phi}_{r, s}^{d}
\end{array}\right\}=\mathbf{T}_{I I R S} \boldsymbol{\Phi}_{r, m}^{d}
$$

where $\mathbf{T}_{I I R S}$ is the appropriate transformation matrix calculated as in references [5, 29, 30]; $\boldsymbol{\Phi}_{r, m}^{d}$ is the $r$-th mode shape that includes the master (denoted by $m$ ) DOFs of damaged structure and $\boldsymbol{\Phi}_{r, s}^{d}$ is the $r$-th mode shape that includes the slave (denoted by $s$ ) DOFs of damaged structure.

From Eqs. (3) and (4), the change ratio of element MKE that can be adopted as a damage indicator is defined by

$$
\mathrm{MKECR}_{e r}=\frac{\mathrm{MKE}_{e r}^{d}-\mathrm{MKE}_{e r}}{\mathrm{MKE}_{e r}}
$$

\subsection{First-order sensitivity calculation of MKE}

The purpose of sensitivity analysis is to evaluate the relationship between dynamic characteristics changes and model's properties changes. By making the first-order derivative of Eq. (3), the sensitivity calculation of MKE with respect to the structural physical parameter $p$ is presented as [24]

$$
\begin{aligned}
& \frac{\partial \mathrm{MKE}_{e r}}{\partial p}
\end{aligned}
$$

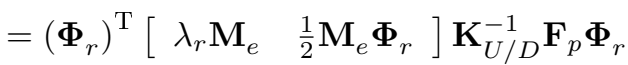

where

$$
\begin{aligned}
\mathbf{K}_{U / D} & =\left[\begin{array}{cc}
\mathbf{K}-\lambda_{r} \mathbf{M} & -\mathbf{M} \boldsymbol{\Phi}_{r} \\
-\left(\mathbf{\Phi}_{r}\right)^{\mathrm{T}} \mathbf{M} & 0
\end{array}\right] \\
\mathbf{F}_{p} & =\left[\begin{array}{c}
-\left(\frac{\partial \mathbf{K}}{\partial p}-\lambda_{r} \frac{\partial \mathbf{M}}{\partial p}\right) \\
\frac{1}{2}\left(\mathbf{\Phi}_{r}\right)^{\mathrm{T}} \frac{\partial \mathbf{M}}{\partial p}
\end{array}\right]
\end{aligned}
$$

The detailed derivations of Eq. (7) can be found in References [24,31]. Herein, assuming that the 
influence of alteration between the mass distribution of the baseline and damaged structures is disregarded. For the sake of simplicity, the $i$ th element has a corresponding design variable named " $p_{i}$ " which is also assumed to be independent of each other. Due to these simplifying assumption, the design variable " $p_{i}$ " is here considered to be the Young's modulus. Consequently, Eq. (7) results in the closed-form sensitivity matrix of MKE as below [24]:

$$
\begin{aligned}
& \frac{\partial \mathrm{MKE}_{e r}}{\partial p}= \\
& \left(\boldsymbol{\Phi}_{r}\right)^{\mathrm{T}}\left[\begin{array}{ll}
\lambda_{r} \mathbf{M}_{e} & \left.\frac{1}{2} \mathbf{M}_{e} \boldsymbol{\Phi}_{r}\right] \mathbf{K}_{U / D}^{-1} \mathbf{F}_{E_{i}} \boldsymbol{\Phi}_{r}
\end{array}\right.
\end{aligned}
$$

where

$$
\mathbf{F}_{E_{i}}=\left[\begin{array}{c}
-\left(\frac{\partial \mathbf{K}}{\partial E_{i}}\right) \\
0
\end{array}\right]
$$

The variation in element MKE and structural stiffness matrix can be determined based on the first-order Taylor's series as follows

$$
\begin{aligned}
\Delta \mathrm{MKE}_{e r} & =\sum_{i}^{N e} \frac{\partial \mathrm{MKE}_{e r}}{\partial E_{i}} \Delta E_{i} ; \\
\Delta \mathbf{K} & =\sum_{i}^{N e} \frac{\partial \mathbf{K}}{\partial E_{i}} \Delta E_{i}
\end{aligned}
$$

where $\Delta \mathbf{K}$ is the overall changes in the stiffness matrix of the structure due to structural deterioration, which is expressed in the form of

$$
\Delta \mathbf{K}=\mathbf{K}-\mathbf{K}^{d}=\sum_{i=1}^{N e} a_{i} \mathbf{K}_{i}
$$

In Eq. (13), the parameter $\alpha_{i}\left(0 \leqslant \alpha_{i} \leqslant 1\right)$ represents the reduction of $i$-th element stiffness due to damage. Substituting Eqs. (12) and (13) into Eq. (10), it yields

$$
\begin{aligned}
\Delta \mathrm{MKE}_{e r}= & \left(\boldsymbol{\Phi}_{r}\right)^{\mathrm{T}}\left[\begin{array}{ll}
\lambda_{r} \mathbf{M}_{e} & \frac{1}{2} \mathbf{M}_{e} \boldsymbol{\Phi}_{r}
\end{array}\right] \\
& \times \mathbf{K}_{U / D}^{-1}\left[\begin{array}{c}
\sum_{i=1}^{N e} a_{i} \mathbf{K}_{i} \\
0
\end{array}\right] \boldsymbol{\Phi}_{r}
\end{aligned}
$$

Finally, by letting Eq. (6) be equal to Eq. (14), one can get

$$
\begin{aligned}
& \underbrace{\mathrm{MKECR}_{e r}}_{\left\{\Delta \mathbf{R}_{e r}\right\}}
\end{aligned}
$$

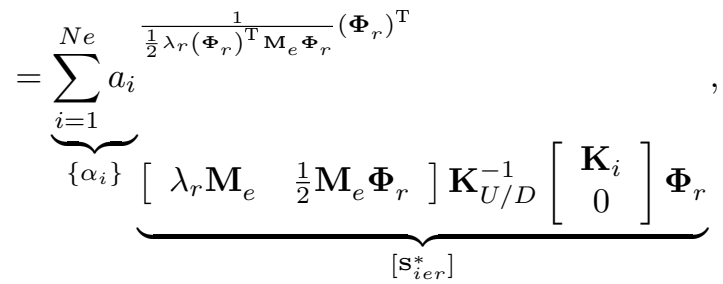

This equation can be rewritten as

$$
\left[\mathbf{S}_{i e r}^{*}\right]\left\{\alpha_{i}\right\}=\{\Delta \mathbf{R}\}_{e r}
$$

where

$$
\left\{\alpha_{\mathbf{i}}\right\}=\left\{\begin{array}{c}
\alpha_{1} \\
\vdots \\
\alpha_{i} \\
\vdots \\
\alpha_{\text {nele }}
\end{array}\right\}
$$

and $\left[\mathbf{S}_{i e r}^{*}\right], \Delta \mathbf{R}$ defined by

$\left[\mathbf{S}_{i e r}^{*}\right]=\left[\begin{array}{ccccc}S_{1,1,1} & \ldots & S_{e, i, r} & \ldots & S_{1, \text { nele }, r} \\ \vdots & & \vdots & & \vdots \\ S_{e, 1, r} & \ldots & S_{e, i, r} & & S_{e, \text { nele }, r} \\ \vdots & & \vdots & & \vdots \\ S_{n e l e, 1, r} & & S_{e, i, r} & & S_{\text {nele }, \text { nele }, r}\end{array}\right]$

$$
\{\Delta \mathbf{R}\}_{e r}=\left\{\begin{array}{c}
\Delta R_{1, r} \\
\vdots \\
\Delta R_{e, r} \\
\vdots \\
\Delta R_{\text {nele }, r}
\end{array}\right\}
$$

are, respectively, the sensitivity matrix and the residual vector.

As can be observed in Eq. (16), the values of vector $\alpha_{i}$ that contains the information of damage location and magnitude can be directly found by solving the above equation set. Because the value of damage is always a non-negative number $\left(\alpha_{i} \geqslant 0\right)$, the non-negative least-squares (NNLS) technique [32] is considered to be particularly appropriate for this purpose. 

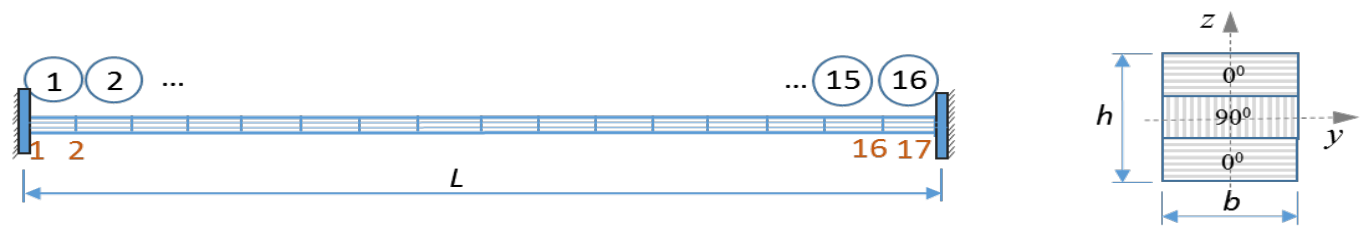

(a)

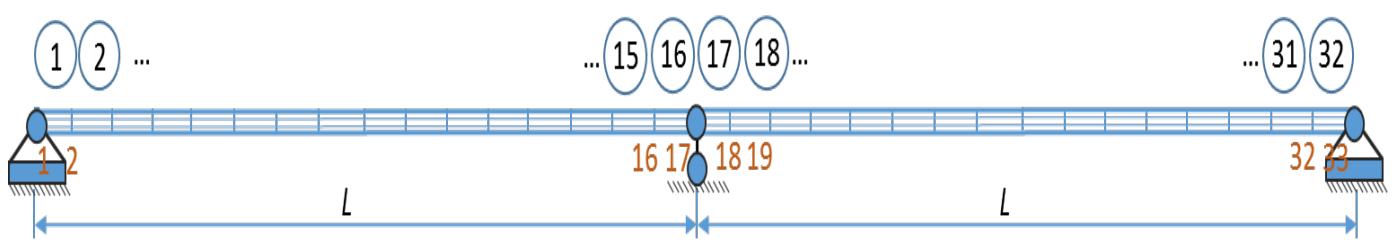

(b)

Fig. 1.(a) Clamped-clamped cross-ply $\left(0^{\circ} / 90^{\circ} / 0^{\circ}\right)$ beam, (b) Two-span continuous cross-ply $\left(0^{\circ} / 90^{\circ} / 0^{\circ}\right)$ beam.

Table 1. Three hypothetical damage scenarios in each composite beam.

\begin{tabular}{cccc}
\hline & Scenarios & Description & $\begin{array}{c}\text { Damaged elements } \\
\text { (reduction of stiffness) }\end{array}$ \\
\hline $\begin{array}{c}\text { Clamped-clamped cross-ply } \\
\left(0^{\circ} / 90^{\circ} / 0^{\circ}\right) \text { beam }\end{array}$ & A & Single damage & $1(10 \%)$ \\
& B & Double damage & $5(10 \%) \& 16(15 \%)$ \\
& C & Multi-damage & $1(10 \%) \& 2(20 \%) \& 5(10 \%)$ \\
& & Double damage & $8(15 \%) \& 25(25 \%)$ \\
\hline Two span continuous cross-ply & D & & \\
$\left(0^{\circ} / 90^{\circ} / 0^{\circ}\right)$ beam & & Triple damage & $7(20 \%) \& 8(15 \%) \& 32(20 \%)$ \\
& E & Multi-damage & $1(15 \%) \& 7(20 \%) \& 8(30 \%)$ \\
& & & $\& 22(30 \%) \& 23(20 \%)$ \\
\hline
\end{tabular}

\section{NUMERICAL EXAMPLE}

In this part, the performance of the presented method for structural damage assessment is investigated through two composite beam structures. The first one is a clamped-clamped crossply $\left(0^{\circ} / 90^{\circ} / 0^{\circ}\right)$ rectangular beam, which is discretized into 16 beam elements using the first- order shear deformation theory (FSDT) [33]. The design of the composite beam is given as follows: the length $L=0.2 \mathrm{~m}$, width $b=0.02 \mathrm{~m}$ and thickness $h=0.02 \mathrm{~m}$, as depicted in Fig. 1(a). The second one is a two-span continuous cross-ply $\left(0^{\circ} / 90^{\circ} / 0^{\circ}\right)$ rectangular beam with the same section, which consists of 32 beam elements as shown in Fig. 1(b). The material properties of both the structures are particularly given as: $E_{1}=40 \mathrm{~N} / \mathrm{m}^{2}, E_{2}=1 \mathrm{~N} / \mathrm{m}^{2}$, 


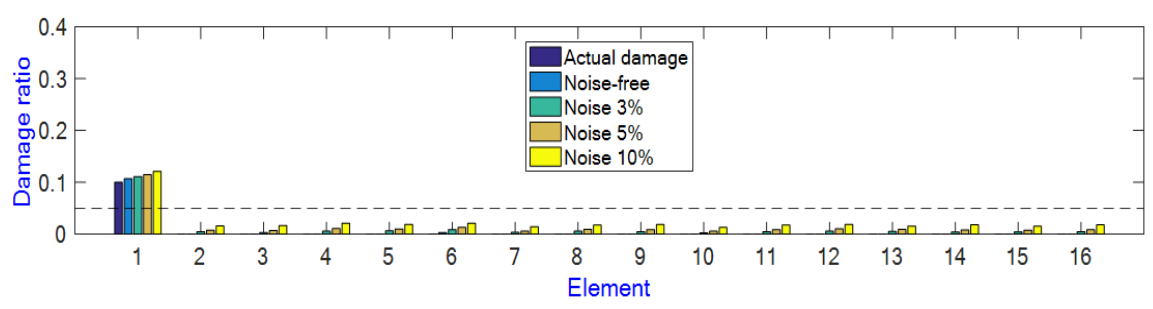

(a)



(b)



(c)

Fig. 2. Damage identification results obtained by damage indicator MKECR for three hypothetical damage scenarios of the clamped-clamped cross-ply $\left(0^{\circ} / 90^{\circ} / 0^{\circ}\right)$ beam:

(a) Scenario A; (b) Scenario B; (c) Scenario C.

$G_{23}=0.5 E_{2}, G_{12}=G_{13}=0.6 E_{2}, v_{12}=0.25$, and the thickness of each layer is $h / 3$. The selection of hypothetical damage scenarios should consider single and multiple damage sites. Table $\mathrm{rgb}] 0.0,0.0,1.01$ presents three different types of damage scenarios imposed on each composite beam.

The modal data of the first five vibration modes are utilized for damage detection in all scenarios. Owing to practical difficulties faced in measuring rotational DOFs, we assume that for both composite beams the used first five mode shapes only involve translational DOFs. In addition to this, these incomplete vibration modes will be polluted with various noise intensities. Particularly, the added noise level of the natural frequencies is fixed at $0.5 \%$, while three noise levels of mode shapes are contaminated with $3 \%, 5 \%$ and $10 \%$. To investigate the influence of noise on damage identification results, 1000 Monte-Carlo simulation runs for each noise level are conducted.

The mean damage values calculated by the MKECR for all damage scenarios of clampedclamped composite beam and two-span continuous composite beam are provided in Fig. 2 and Fig. 3, respectively. By setting a threshold value of 0.05 , all members that exceed this threshold value, are considered as damaged members. Overall, it is apparent that for the cases of noisefree and noise levels $(3 \%, 5 \% 10 \%)$, the proposed method can correctly determine the dam- 


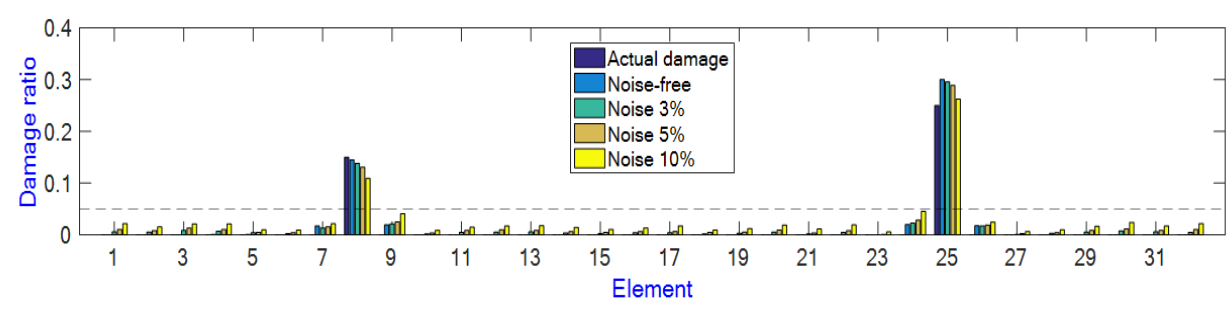

(a)

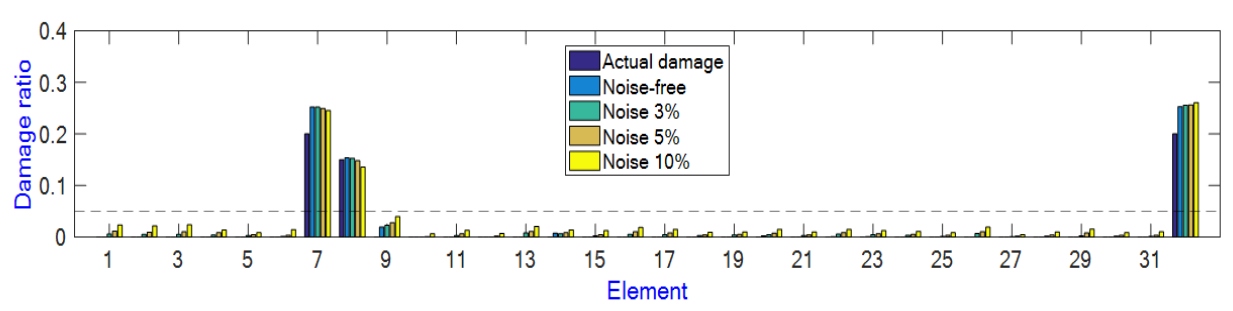

(b)

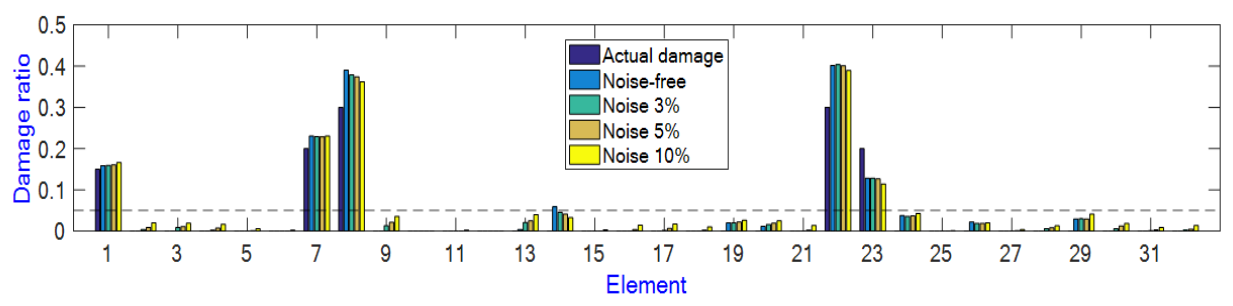

(c)

Fig. 3. Damage identification results obtained by damage indicator MKECR for three hypothetical damage scenarios of the two-span continuous cross-ply $\left(0^{\circ} / 90^{\circ} / 0^{\circ}\right)$ beam: (a) Scenario D; (b) Scenario E; (c) Scenario F.

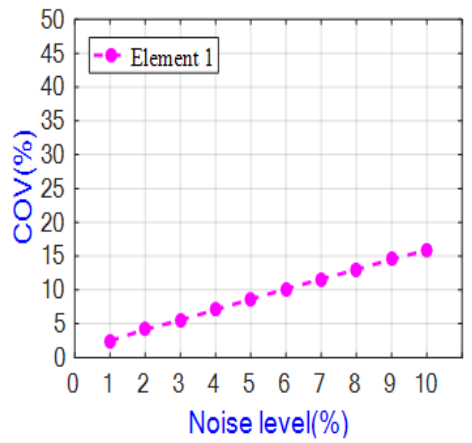

(a)



(b)



(c)

Fig. 4. Coefficients of variances for damaged elements in the case of different noise levels: (a) Scenario A; (b) Scenario B; (c) Scenario C. 




(a)

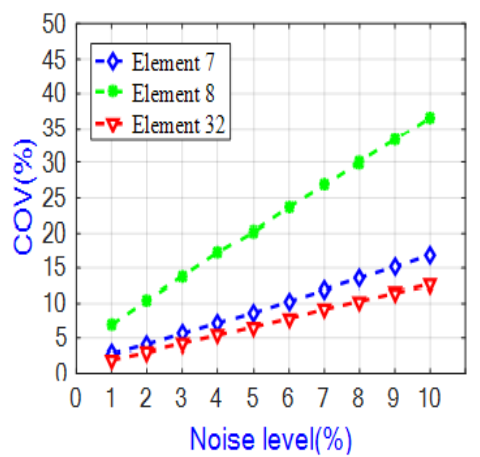

(b)

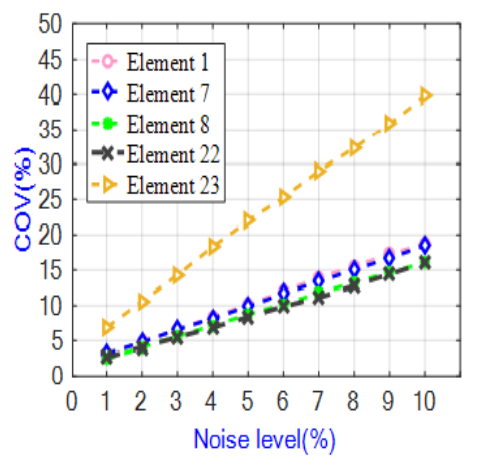

(c)

Fig. 5. Coefficients of variances for damaged elements in the case of different noise levels: (a) Scenario D; (b) Scenario E; (c) Scenario F.

aged site(s) in all presumed damage scenarios of both beam structures. As to damage quantification, the average estimates of elemental stiffness factors obtained by the MKECR are relatively close to the actual values, except for a few damage locations in multiple damage cases $\mathrm{C}$ and F. Also from the figures, one can see that in most cases, the trends in prediction errors increase with increasing noise intensity, but not significantly. For further investigation, the coefficient of variance (COV) is then used to better understand the uncertainties involved in damage severity estimation. The COV, a unit-less measure, is defined as the ratio of the standard deviation $\left(\bar{\sigma}_{\alpha}\right)$ to the average value $\left(\bar{m}_{\alpha}\right)$

$$
\operatorname{COV}(\%)=\frac{\bar{\sigma}_{\alpha}}{\bar{m}_{\alpha}} \times 100
$$

Fig. 4 and Fig. 5 show the COV values for damaged elements in all damage scenarios of clamped-clamped composite beam and two-span continuous composite beam under noise conditions, respectively. From these figures, one can see that:

(1) The COV values linearly increase with adding noise levels.

(2) The parameter COV is also affected by two factors: the position of damage and its magnitude. For example, the values of $\mathrm{COV}$ for element 5 (scenarios B and C) and 8 ((scenarios $\mathrm{D}$ and $\mathrm{E}$ ) are much larger than that of others. Meanwhile, the value of $\mathrm{COV}$ for element 8 (scenarios $\mathrm{F}$ ) is considerably decreased as its damage extent (scenarios F) increases.

(3) The damage prediction at damaged locations that has larger $\mathrm{COV}$ in comparison with others generally exhibits high uncertainty.

Furthermore, the influence of used number of mode shapes, as well as damage magnitudes on the results of damage detection, is successively investigated. For this purpose, scenario A with $10 \%$ noise imposed on the first five mode shapes is utilized in damage extent estimation. Referring to Fig. 6, it is apparent that the parameter COV for damaged element 1 decreases with increasing the magnitude of damage. Fig. 7 indicates that this parameter is also dependent on the number of modes employed in damage extent estimation. Among different vibration modes (first 3, 4 and 5modes) examined in the scenario, the smallest value of the parameter COV is always found when the first 5 modes are considered for damage identification.

For the purpose of investigating the influence of fiber orientation angles in a composite structure on the accuracy of the proposed damage identification method, a clamped-clamped composite $\left(\theta^{0} /-\theta^{0} / \theta^{0}\right)$ beam with various fiber orientation angles $\theta=15^{\circ}$ to $90^{\circ}$ is studied. The composite $\left(\theta^{0} /-\theta^{0} / \theta^{0}\right)$ beam has the same geometric and material properties as in the first 


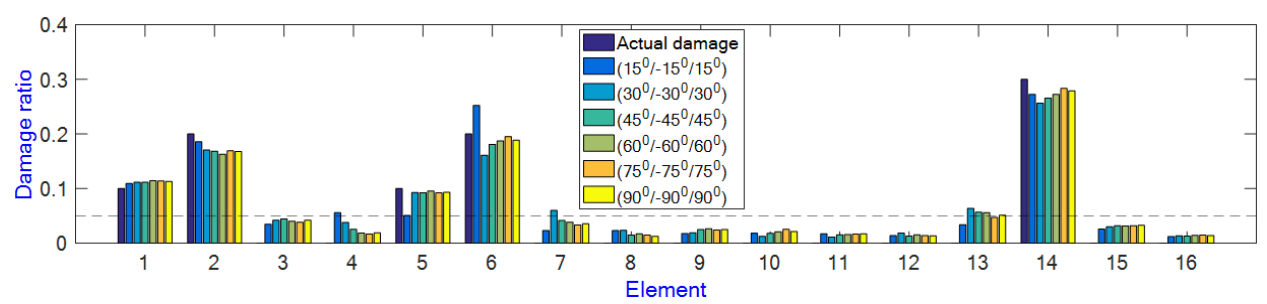

Fig. 8. Damage identification results obtained by damage indicator MKECR for damage scenario $\mathrm{C}$ of the clamped-clamped cross-ply $\left(\theta^{0} /-\theta^{0} / \theta^{0}\right)$ beam with various fiber orientation angles.



Fig. 6. Coefficients of variances for damaged element 1 in scenario A with three different damage degrees $(10 \%, 20 \%$ and $30 \%)$.

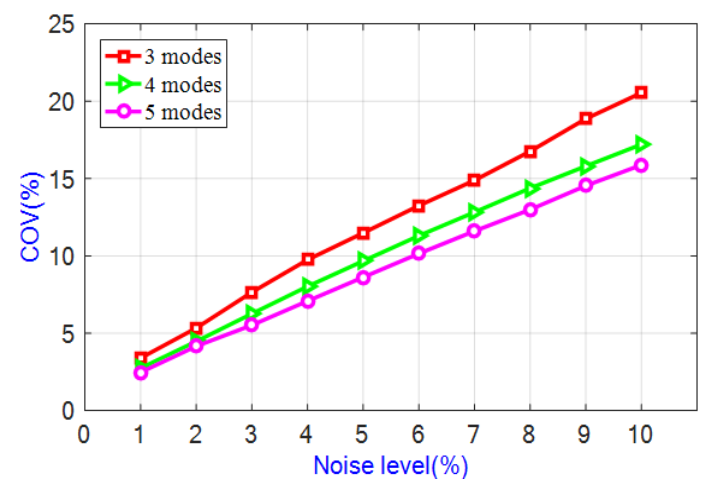

Fig. 7. Coefficients of variances for damaged element 1 in scenario A, using different vibration modes (first 3, 4 and 5 modes considered). beam. Here, damage situation is assumed to be same as scenario C in Table rgb]0.0,0.0,1.01. For the purpose of damage identification in this composite beam, the information on the first five incomplete modes with noise level at $10 \%$ is utilized. According to Fig. 8, the arrangement of fiber orientation angles has less influence on the damage identification results. It is clearly seen that for all the orientation angles, the average identified damage severities are relatively close to the real values, although the proposed method has several false alarms elements with small severities appeared in its predictions.

\section{CONCLUSIONS}

The article aims to explore a good damage sensitive parameter termed modal kinetic energy change ratio (MKECR) to damage localization and quantification of laminated composite beams. Numerical simulations are conducted on a clamped-clamped composite beam and a two-span continuous composite beam with various hypothetical damage scenarios to investigate the performance of the proposed damage diagnosis method based on MKECR. Special attention is paid to the effect of different noise levels on the precision of damage assessment results. To gain the statistical results of damage assessment, 1000 Monte Carlo simulation runs are carried out for each case under investigation. Based on the numerical results above, it is possible to conclude that even under incomplete measurements and high measurement noise, the proposed method can reliably identify the true damage locations. Although in some cases, the 
prediction of damage magnitudes at damaged locations are not close to the true values, the results are acceptable at a practical level. Besides, the statistical analysis also confirmed that the quality of the damage prediction results depends on the quality of measured vibration data, the number of used modes as well as damage magnitudes. Despite this, experimental investigations are still needed to provide further insight into how well the proposed damage identification method performs under varying operational and environmental factors.

\section{ACKNOWLEDGEMENT}

This research is funded by Vietnam National Foundation for Science and Technology Development (NAFOSTED) under grant number 107.02-2017.08.

\section{References}

[1] Montalvao, D. (2006). A Review of Vibration-Based Structural Health Monitoring with Special Emphasis on Composite Materials, The Shock and Vibration Digest, 38(4), 295-324.

[2] Gomes, G. F., Alí, Y., Mendéz, D., Lopes, S., Simões, S., Carlos, A., \& Jr, A. (2018). The Use of Intelligent Computational Tools for Damage Detection and Identification with an Emphasis on Composites - A Review, Composite Structures, 196(May), $44-54$.

[3] Vosoughi, a. R., \& Gerist, S. (2014). New Hybrid FE-PSO-CGAs Sensitivity Base Technique for Damage Detection of Laminated Composite Beams, Composite Structures, 118, 68-73.

[4] Gomes, G. F., da Cunha, S. S., \& Ancelotti, A. C. (2018). A Sunflower Optimization (SFO) Algorithm Applied to Damage Identification on Laminated Composite Plates, Engineering with Computers, 0(0), 0.
[5] Dinh-Cong, D., Dang-Trung, H., \& Nguyen-Thoi, T. (2018). An Efficient Approach for Optimal Sensor Placement and Damage Identification in Laminated Composite Structures, Advances in Engineering Software, 119, 48-59.

[6] Dinh-Cong, D., Vo-Duy, T., Nguyen-Minh, N., Ho-Huu, V., \& Nguyen-Thoi, T. (2017). A Two-Stage Assessment Method Using Damage Locating Vector Method and Differential Evolution Algorithm for Damage Identification of Cross-Ply Laminated Composite Beams, Advances in Structural Engineering, 20(12), 1807-1827.

[7] Dinh-Cong, D., Vo-Duy, T., Ho-Huu, V., \& Nguyen-Thoi, T. (2019). Damage Assessment in Plate-like Structures Using a Two-Stage Method Based on Modal Strain Energy Change and Jaya Algorithm, Inverse Problems in Science and Engineering, 27(2), 166-189.

[8] Kumar, M., Shenoi, R. A., \& Cox, S. J. (2009). Experimental Validation of Modal Strain Energies Based Damage Identification Method for a Composite Sandwich Beam, Composites Science and Technology, 69(10), 1635-1643.

[9] Kim, J. T., Ryu, Y. S., Cho, H. M., \& Stubbs, N. (2003). Damage Identification in Beam-Type Structures: Frequency-Based Method vs Mode-Shape-Based Method, Engineering Structures, 25(1), 57-67.

[10] Alvandi, A., \& Cremona, C. (2006). Assessment of Vibration-Based Damage Identification Techniques, Journal of Sound and Vibration, 292(1-2), 179-202.

[11] Li, Y. Y. (2010). Hypersensitivity of StrainBased Indicators for Structural Damage Identification: A Review, Mechanical Systems and Signal Processing, 24(3), 653-664.

[12] Stubbs, N.; Kim, J. T.; Farrar, C. R. (1995). Field Verification of a Nondestructive Damage Localization and Severity Estimation Algorithm, Proceedings of the 13th International Modal Analysis Conference (IMAC XIII), 182, 210-218. 
[13] Stubbs, N., \& Kim, J.-T. (1996). Damage Localization in Structures without Baseline Modal Parameters, AIAA Journal, 34(8), 1644-1649.

[14] Shi, Z. Y., Law, S. S., \& Zhang, L. M. (1998). Structural Damage Localization From Modal Strain Energy Change, Journal of Sound and Vibration, 218(5), 825-844.

[15] Hu, S. J., Wang, S., \& Li, H. (2006). CrossModal Strain Energy Method for Estimating Damage Severity, Journal of Engineering Mechanics, 132(4), 429.

[16] Guo, H. Y., \& Li, Z. L. (2014). Structural Multi-Damage Identification Based on Modal Strain Energy Equivalence Index Method, International Journal of Structural Stability and Dynamics, 14(07), 1450028 .

[17] Seyedpoor, S. M. (2012). A Two Stage Method for Structural Damage Detection Using a Modal Strain Energy Based Index and Particle Swarm Optimization, International Journal of Non-Linear Mechanics, $47(1), 1-8$.

[18] Li, Y., Zhang, M., \& Yang, W. (2018). Numerical and Experimental Investigation of Modal-Energy-Based Damage Localization for Offshore Wind Turbine Structures, Advances in Structural Engineering, 21(10), $1510-1525$.

[19] Hu, H., Wang, B.-T., Lee, C.-H., \& Su, J.-S. (2006). Damage Detection of Surface Cracks in Composite Laminates Using Modal Analysis and Strain Energy Method, Composite Structures, 74(4), 399-405.

[20] Pradeep, K. R., Rao, B. N., Srinivasan, S. M., \& Balasubramaniam, K. (2014). Modal Strain Energy Change Ratio for Damage Identification in Honeycomb Sandwich Structures, Canadian Journal of Basic and Applied Sciences, 02(01), 10-24.

[21] Vo-Duy, T., Ho-Huu, V., Dang-Trung, H., Dinh-Cong, D., \& Nguyen-Thoi, T. (2016). Damage Detection in Laminated Composite Plates Using Modal Strain Energy and
Improved Differential Evolution Algorithm, Procedia Engineering, 142(April), 181-188.

[22] Yan, W., Huang, T., \& Ren, W. (2010). Damage Detection Method Based on Element Modal Strain Energy Sensitivity, Advances in Structural Engineering, 13(6), 1075-1088.

[23] Entezami, A., \& Shariatmadar, H. (2014). Damage Detection in Structural Systems by Improved Sensitivity of Modal Strain Energy and Tikhonov Regularization Method, International Journal of Dynamics and Control, 2(4), 509-520.

[24] Hadjian Shahri, A. H., \& Ghorbani-Tanha, A. K. (2017). Damage Detection via ClosedForm Sensitivity Matrix of Modal Kinetic Energy Change Ratio, Journal of Sound and Vibration, 401, 268-281.

[25] Dinh-Cong, D., Nguyen-Thoi, T., Vinyas, M., \& Nguyen, D. T. (2019). Two-Stage Structural Damage Assessment by Combining Modal Kinetic Energy Change with Symbiotic Organisms Search, International Journal of Structural Stability and Dynamics, 1950120.

[26] Meng, H. P., \& Lin, X. Y. (2012). Damage Orientation Method Based on the Modal Kinetic Energy, Applied Mechanics and Materials, 256-259, 1112-1116.

[27] O'Callahan, J. C. (1989). A Procedure for an Improved Reduced System (IRS) Model, Proceedings of the 7th International Modal Analysis Conference, 17-21.

[28] Dinh-Cong, D., Pham-Duy, S., \& NguyenThoi, T. (2018). Damage Detection of 2D Frame Structures Using Incomplete Measurements by Optimization Procedure and Model Reduction, Journal of Advanced Engineering and Computation, 2(3), 164-173.

[29] Friswell, M. I., Garvey, S. D., \& Penny, J. E. T. (1995). Model Reduction Using Dynamic and Iterated IRS Techniques, Journal of Sound and Vibration, 186(2), 311-323.

[30] Dinh-Cong, D., Pham-Toan, T., NguyenThai, D., \& Nguyen-Thoi, T. (2019). Structural Damage Assessment with Incomplete 
and Noisy Modal Data Using Model Reduction Technique and LAPO Algorithm, Structure and Infrastructure Engineering, 15(11), 1436-1449.

[31] Lee, I.-W., \& Jung, G.-H. (1997). An Efficient Algebraic Method for the Computation of Natural Frequency and Mode Shape Sensitivities - Part I. Distinct Natural Frequencies, Computers \& Structures, 62(3), 429-435.

[32] Lawson, C. L., \& Hanson, R. J. (1995). Solving Least Squares Problems, Siam.

[33] Vo-Duy, T., Nguyen-Minh, N., DangTrung, H., Tran-Viet, A., \& Nguyen-Thoi, T. (2015). Damage Assessment of Laminated Composite Beam Structures Using Damage Locating Vector (DLV) Method, Journal of Contemporary Physics, 50(4), 457-465.

\section{About Authors}

Du DINH-CONG was born in Binh Dinh, Vietnam, 1987. He received the B.Sc. and MSc degree in Civil Engineering from Ho Chi Minh City Open University, Vietnam in 2011 and 2014, respectively. Currently, he is pursuing his Ph.D. degree in Computational Science and works at Institute for Computational Science (INCOS), Ton Duc Thang University. His main research activities include structural health monitoring, optimization algorithms, model-order reduction techniques and interval finite element methods.
Dung NGUYEN-QUOC was born in Binh Dinh, Vietnam in 1970. He received the B.Sc. in Civil Engineering and Industry at Da Nang University of Technology, Vietnam in 2004. Currently, he is pursuing MSc degree in Civil Engineering, Ton Duc Thang University and works at Binh Dinh Economic Zone Authority, Vietnam. His research is within the fields of structural health monitoring and optimization algorithms.

Linh VO-VAN was born in Binh Dinh, Vietnam, in 1978. He received the B.Sc. degree in Civil Engineering from Van Lang University, Vietnam in 2001. Currently, he is pursuing his M.Sc degree in Civil Engineering, Ton Duc Thang University. He is also the vice-director of Project Management and Ground Clearance at Binh Dinh Economic Zone Authority, Vietnam. His research interests are in the areas of structural health monitoring and optimization algorithms.

Trung NGUYEN-THOI received the Ph.D degree in Mechanical Engineering, National University of Singapore (NUS), Singapore in 2010. He was appointed as a full professor of Ton Duc Thang University, Vietnam in 2017. Currently, he is director of Institute for Computational Science (INCOS), Ton Duc Thang University. His research interests include computational mechanics, reliability analysis of structures, structural health monitoring, optimization algorithms, intelligent computation, structural optimization and applied optimization. 